\title{
A brand new cheating attempt: a case of usurped identity
}

\author{
Yves Crama $^{1}$ - Michel Grabisch ${ }^{2}$. \\ Silvano Martello ${ }^{3}$
}

Published online: 5 October 2016

(C) Springer-Verlag Berlin Heidelberg 2016

\begin{abstract}
We recall some cases of ethical misconduct that occurred in the recent years, and we report a new trick that was recently attempted to cheat the journal in order to obtain the publication of an article through usurped identity.
\end{abstract}

\section{Plagiarism and other tricks}

Since its foundation in 2003, $4 O R$ has experienced plagiarism and cheating attempts. In all cases, our policy has been to ban the "authors" from submitting to the journal and to publicize the facts through editorials. We believe indeed that publishing an account on these highly un-ethical behaviors is a deterrent for future attempts and provides useful information to the editors of scientific journals. In fact, according to our own experience, plagiarists and cheaters do not stop their fraudulent activity as soon as they are caught by a journal, but keep trying with other journals. We thus believe it is useful, before describing the latest attempt we faced, to briefly recall the most relevant situations we encountered in the past. More details, with copies of the original

Silvano Martello

dipdeis.4or@unibo.it

Yves Crama

Yves.Crama@ulg.ac.be

Michel Grabisch

michel.grabisch@univ-paris1.fr

1 HEC Liège, Université de Liège, Rue Louvrex 14 (N1), 4000 Liège, Belgium

2 Centre d'économie de la Sorbonne, Université Paris I, 106-112, Boulevard de 1'Hôpital, 75013 Paris, France

3 DEI “Guglielmo Marconi”, Università di Bologna, Viale Risorgimento 2, 40136 Bologna, Italy 
documents, can be found at the journal home page http://www.4or.be/Plagiarism.html, where the complete list of the banned authors is also provided.

The most famous case we faced was also the first one: in 2002, during the preparation of the first 4OR issues, Dănuţ Marcu from Bucharest submitted a short paper on the independence number of a graph. The refereeing process showed that it was a very slightly modified copy of a paper published a few years earlier by J.B. Shearer in the Electronic Journal of Combinatorics. In addition, a thorough investigation through the Internet highlighted an impressive number of plagiarized papers published by Marcu (and, in most cases, retracted by the journal editors once the fact was discovered). Detailed accounts on these facts can be found in the $4 O R$ Editorials Bouyssou et al. (2006) and Bouyssou et al. (2009), as well as in an investigation conducted by the journal Geombinatorics, see Soifer (2007). Marcu became so famous that he has now a dedicated Wikipedia page and, to the best of our knowledge, he terminated his fraudulent activity.

In 2007 M. Sreenivas and T. Srinivas submitted to $4 O R$ a manuscript on the transportation paradox, that turned out to be almost identical to a paper published in Optimization Online by S. Storøy. When caught, they sent an email of profound excuses, but, at the same time, they submitted the same manuscript to the European Journal of Operational Research (where the plagiarism was also detected). The investigations conducted by $4 O R$ and reported in the editorial Bouyssou et al. (2009), highlighted other misconducts. The editorial was followed by an investigation of SIAM (see http://www.siam.org/journals/plagiary/index.php), and to the retraction of articles published by the International Journal of Statistics and Management System (see http://anson.ucdavis.edu/ jiang/IJSMS/3-10-retraction.html) and by RAIRO [see their editorial Mahey (2010)]. Although Sreenivas and Srinivas are not as famous as Marcu, if one searches for their names on the Internet, evidence of their misconduct immediately shows up.

In recent years, most publishers adopted plagiarism detection tools to pre-process all submissions, which made the plagiarists life less easy. However, other methods have been developed that threaten the journals' scientific integrity, including hacking the electronic submission systems, tricking the refereeing process, or selling the authorship of accepted publications, as reported in the recent 4OR editorial Liberti et al. (2015). A new trick that was recently attempted is reported in the next section.

\section{The new case: usurped identity}

A few months ago, a manuscript authored by a well-known Dutch economist was submitted to 4OR using a Yahoo $\odot$ email address. Having some doubt, the handling Editor-in-Chief wrote to the author's institutional address

Since some journals have recently been spammed by fake submissions, since this particular paper has been submitted from a Yahoo.com email address, and since it does not seem to match your usual field of research, I would appreciate if you could confirm that you are indeed the author of this submission before I process it further. 
The reply was immediate:

Thank you for getting in touch with me! I'm most certainly NOT the author of that article and, indeed, this is the third time this year someone has submitted an article in my name using this email address. I don't know what to do about this personally, other than hope that all editors are as vigilant as you are, for which you, again, have my thanks.

Upon consulting the Springer Director of Publishing Integrity, we decided to play the game in order to understand the objective of this strange behavior. We pretended that we were processing the submission normally and we assigned it to ourselves as (anonymous) referees. After a couple of months, we prepared fake reviews and pretended that the manuscript was going to be accepted for publication subject to minor revisions. The decision was communicated to the alleged author through the electronic submission system.

The revised manuscript came quite quickly, but this time the authors were Alireza Noruzi, Hasan Jafari (both with affiliation Young Researchers and Elite Club, Ardabil Branch, Islamic Azad University, Ardabil, Iran), Tohid Banki (Department of Engineering, Bilesuvar Moghan Branch, Islamic Azad University, Bilesuvar, Moghan, Iran), and Mohsen Mohammadi (Department of Electrical Engineering, Payame Noor University (PNU), Tehran, Iran), while the name of the Dutch economist had disappeared. We then wrote to the Yahoo address

Dear Dr. Noruzi,

Thank you for submitting the revised version of your manuscript entitled "DayAhead Price Forecasting of Electricity Markets by Fuzzy Chaotic Honey Bee Mating Optimization, Wavelet Transform and Feature Selection" to $4 O R-A$ Quarterly Journal of Operations Research.

I have noticed that you are now listed as corresponding author in the system, together with Hasan Jafari, Tohid Banki, and Mohsen Mohammadi, whereas $\mathrm{XXX}$ was listed as the corresponding author in the original manuscript.

As mentioned in our previous correspondence, the journal's policy is that: "Any variation in the authors list must be highlighted to the Editors-in-Chief: It is at their discretion as to whether these changes are allowed."

Kindly confirm who will be the corresponding author so that all correspondence regarding the submission can be addressed to the correct person.

and the answer was

Dear Professor,

Many thanks for your kind email. Regarding to the mentioned problem, I have to say that XXX was the submission responsible of our paper. Where, after revision he doesn't like to be as an author in this manuscript. Furthermore, according to some strict sanctions we obligated to do this. Anyway I apologize for this problem which provides some misunderstanding.

Thanks again. All the best,

Authors 
At this point, the objective was clear (avoid desk rejection and count on the reputation of the well known pretended author to ease acceptance), so we decided to stop playing cat and mouse. We wrote to the real authors that we had discovered this extreme case of dishonest scientific conduct, coupled with a case of usurpation of identity, that they are indefinitely banned from publishing in 4OR, and that their names will be publicized in an editorial.

We hope that this account will stop the fraudulent activity of these individuals. It is clear however that Internet addresses can be a useful tool in the hands of dishonest persons, so future attempts of this kind cannot be excluded. We believe that scientific journals should evaluate which actions could help to preserve their integrity.

\section{References}

Bouyssou D, Martello S, Plastria F (2006) A case of plagiarism: Dănuţ Marcu. 4OR 4(1):11-13

Bouyssou D, Martello S, Plastria F (2009) Plagiarism again: Sreenivas and Srinivas, with an update on Marcu. 4OR 7(1):17-20

Liberti L, Marchant T, Martello S (2015) The dirty dozen of 4OR. 4OR 13(1):1-13

Mahey P (2010) A case of plagiarism: retraction of a paper by Sreenivas and Srinivas. RAIRO Rech Opér 44(1):1-3

Soifer A (2007) The case of Dr. Dănuţ Marcu: serial plagiarism and signing false statements. Geombinatorics XVI(3):293-296 